\title{
Mobile Adhoc Routing Scheme in Reducing Energy Consuming
}

\author{
Jiang Xiaojie \\ Weihai ocean vocational college \\ maggie_jesse@163.com
}

\begin{abstract}
Mobile Ad hoc network, the author of this paper the energy consumption of a larger problem, analyses the reasons of large energy consumption. Mobile Ad Hoc network node has very limited resources, especially energy, through the design of effective power control strategy combined with routing to reduce the network energy consumption is very important and necessary. And as the goal, put forward the improvement strategy, proposed further reduce routing mechanism in mobile Ad-hoc energy costs, simulation and analysis show that the advantages of this solution.
\end{abstract}

Keywords: routing, mobile ad-hoc, energy consumption, route discovery.

\section{Introduction}

Compared with the traditional network, Ad Hoc network nodes generally by exhaustible energy batteries to provide power, in many applications energy limited and not promptly added. Ad Hoc network limited energy has become a bottleneck restricting its extensive application, how to effectively use of the limited energy of nodes has become a problem to be solved in the practical application. In Ad Hoc network node energy consumption can be divided into communication energy cost and the energy cost two parts. Among them: the former refers to the communication related to the energy dissipations of the send and receive and process the data; Which nodes for the sake of communication that is used to maintain normal operation and run the energy dissipations of the network application and agreement, such as data compression, the energy dissipations of the decoding, error correction and so on. In Ad Hoc networks, the node energy is mainly used for sending and receiving data packet, and used in the communication part is limited. Because not only depends on the application and agreement and communication energy costs inherent factors such as the specific hardware configuration, in order to effectively reduce the network energy costs, now largely only through energy saving communication overhead.

Many researchers through the power control strategy is presented to reduce the energy consumption of the network. Power control is mainly considering the energy dissipations of the sending and receiving data grouping nodes, it is closely related to many of Ad Hoc network protocol layer, profoundly influenced the physical link quality, the bandwidth of the MAC layer and spatial reuse degree, but also affect the network layer routing, congestion control of the transport layer and application layer business quality [1-4]. The existing power control in Ad Hoc network research mainly focused on three aspects, namely, the network layer of the power control, power control of MAC layer and cross-layer power control. Cross-layer power control between the network layer and MAC layer protocol layer to timely sharing information, integrated power control was carried out on the network to reduce the network energy 
consumption, is a research hotspot of the current power control. Typical Ad Hoc routing protocols, such as the dynamic source routing protocols DSR, purpose sequence distance vector protocols DSDV and on-demand distance vector protocols AODV, usually with the minimum hop count as the basis of choosing path. Although these protocol implementation is easy, but does not take into account the network energy consumption. Mobile Ad Hoc network node has very limited resources, especially energy, through the design of effective power control strategy combined with routing to reduce the network energy consumption is very important and necessary. In view of this, this article will on in-depth research and discussion on this question.

\section{Related Works}

In recent years, based on power control of Ad Hoc routing protocol research more and more get people's attention. In order to reduce the network energy consumption, [5] on the basis of AODV protocol, power factor function is introduced to find the path of the minimum energy consumption. Listen for IEEE 802.11 carrier Multiple Access/Collision Avoidance (CarrierSense Multiple Access with Collision Avoidance, CSMA/CA) protocol does not guarantee to find the minimum energy consumption routing problems, literature [6] based on the AODV protocol, the routing packet forward the introduction of a waiting time to optimize routing, so as to achieve the purpose of reducing energy costs. [7] of the DSR protocol routing discovery process is improved. In the scenario, each node according to residual energy to decide whether to participate in the routing discovery process, thus to prolong network lifetime. In the literature [8], each node maintains several zhang lu4 by table of different sending power level, the node with minimum of the power level of routing, and the unity of the corresponding power to send data. For [9] the uneven distribution of the nodes, the network communication power may be much improved, when forwarding a packet to the destination node, choose the lowest level can reach the destination node power routing, and then to the corresponding power sends it to the next hop node in order to save the network energy consumption. In addition, the document [10] by the data link layer provides the link information, in the use of AODV protocol to find and establish a routing process, by dynamically adjust the transmission power to save the network energy consumption. The literature [11] only takes into account the network layer of the energy costs, does not take into account the corresponding power consumption at the MAC layer, failed to minimize the network energy consumption and literature [12] proposed routing based on cross-layer power control can solve this problem well. [13] based on the distance between the node and sends the power relations, iteratively increased between the source node and destination node relay nodes, with the minimum power to send DATA/ACK packet to save the network energy consumption. [14] based on the multi-hop energy-saving features of wireless transmission, through the establishment of efficient power neighbor set to control the routing request packet forwarding process, so as to achieve the purpose of reduce the network energy costs.

\section{System Model Description}

Power control is a challenging research topic, 1. m. Kirousis and others in the literature [14] in the scope of its modeling for sending allocation (Range the Assignment, RA) problem. Aiming at the computational complexity of the problem, this paper is dedicated to the details of the theoretical proof and simulation analysis. To $r\left(u_{i}\right)$ indicate the node transmission radius $u_{i}(1 \leq i \leq n), N=\left\{u_{1}, L u_{n}\right\}$ said $k$ node 
collection of physical location. RA problem send network power $f\left[r\left(u_{j}\right)\right]$ is expressed as size

$$
f\left[r\left(u_{j}\right)\right]=\sum_{u_{i} \in N}\left[r\left(u_{j}\right)\right]^{\alpha}
$$

Where $2 \leq \alpha \leq 5, \quad k=1,2,3$.

Without destroying the premise of network connectivity, RA problem is $f\left[r\left(u_{j}\right)\right]$ minimized, namely

$$
f\left[r\left(u_{j}\right)\right]_{\min }=\min \sum_{u_{i} \in N}\left[r\left(u_{j}\right)\right]^{\alpha}
$$

In one dimensional case, (2) can be solved in time $O\left(n^{4}\right)$, however, in the case of 2 Dand $3 \mathrm{D},(2)$ is NP - hard problem. In fact, the actual power control problems than RA is more complicated.

The results show that the optimal solution for RA problem not reality. To this, this article embarks from the actual, trying to reduce packet transmission power for RA, practical solutions to achieve the purpose of reduce the network energy consumption. Assume that link the bidirectional symmetric, each node of the maximum send power, the same node sending power $P_{t}$ and received power $P_{r}$ obedience to the power path loss, namely

$$
c P_{t} d^{-\alpha}=P_{r}
$$

Which $c$ is constant, $d$ Euler distance between nodes for sending and receiving. $\alpha$ Ditto, specific value depends on the wireless propagation model, for freedom model $\alpha=2$, the double model $\alpha=4$.

A node receiving data correctly received power $\gamma$, at least for it

$$
\frac{c P_{t}}{d^{\alpha}} \geq \gamma
$$

By a type (4) the

$$
P_{t} \geq \frac{\gamma}{c} d^{\alpha}
$$

In order to effectively support the Ad Hoc network node mobility and save the network energy consumption, simplified network model at the same time, this article only adjust the sending power nodes are different in a number of discrete power level (see definition 2-1). The corresponding support hardware CiscoAironet350 and 1200 CARDS, and other series, 350 series of six power level $(1,5,20,30,50$ and $100 \mathrm{~mW})$, the 1200 series has three power level (5, 10 and $30 \mathrm{~mW}$ ). From expression (3) and (5) it can be seen that: different transmission power level covers different distance node, the search for neighbors is different also.

For the purposes of this paper, the following definition in the paper the concepts involved.

Definition 2 - (1) power level nodes sending power change between the finite discrete values, different power called power level. Power level between a and $b$ is expressed as nodes, node $\mathrm{a}$ and $\mathrm{b}$, the minimum power level is expressed as between 
nodes data grouping and MAC layer between $a$ and $b$ grade control frame of power respectively as to.

Routing rule 1 if the node $u$ has a different power level $k$ route to the destination node $v \quad(p l=i, 1 \leq i \leq k)$ routing $R T_{\min p l}^{(u, v)}$, the choice of power level to the smallest routing $R T_{\min p l}^{(u, v)}$ as its transmitting data packet routing.

Routing rule 2 if the node $u$ has multiple power level $p l$ are the smallest and can reach the destination node $v$ routing $R T_{\min p l}^{(u, v)}$, the select minimum hop $h$ routing as its transmitting data packet routing $R T_{\min p l}^{(u, v)}$.

\section{The Proposed Scheme}

PC - AODV is an on-demand routing protocol, its core idea is: (1) according to the need to build more power level routing, node according to the routing rules 1 and 2 to choose transmit data routing; (2) of different group and MAC frame control different power control measures. PC - AODV routing discovery and routing maintenance divides into two parts. Is already defined in the IEEE standard 802.1 1 of MAC layer control frame mainly include: the RTS and CTS and ACK control frame. PC - AODV grouped according to the data transmission, routing and MAC layer control message frames using different types of different power control strategy to reduce the network energy consumption. Routing messages sent in different power level; For the application of data packet sending power according to the routing table table item Settings; To control the MAC layer frame sending power set according to the routing message sending and application data grouping the power and change the Settings, and to remain consistent.

PC-AODV based on AODV, power control is introduced to optimize the performance of AODV. Algorithm is mainly composed of the following four parts.

Step 1 to determine whether there is a routing; When node has data sent to the destination node, the first route table lookup, if there is a route to the destination node $\mathrm{v}$, perform Step 4, otherwise do Step 2.

Step 2 in different power level to establish to the destination node routing: when there is no node to the destination node routing, respectively in different power level () to the destination node routing, where $\mathrm{n}$ is the total power series. Found in each of the different power level routing process, intermediate nodes only received for the first time with the same < Source ID, Broadcast ID, pl > RREQ (Route REQuste) packet forwarding, and destination node is only for the first time to receive with the same < Source ID, Broadcast ID, pl > RREQ packet REPly RREP (Route REPly) group. In this way, which is more than can be formed by the source node $u$ can reach the destination node $\mathrm{v}$ power levels of different routing $R T_{\min p l}^{(u, v)}$. In any route discovery process, the PC - AODV routing message in a unified power pl send, at the same time adjusting the corresponding MAC layer transmission control send power level $p l_{M A C}$ consistent with its frame, namely

$$
p l_{M A C}=p l
$$


Step 1, 2, 3 according to the routing rule to determine the destination node routing: set $u_{j} \rightarrow v$ said node routing rules 1, 2, in accordance with choice of route to the destination node $v$. Among them: the next-hop node $u_{j+1}$ to node $u_{j}, 0 \leq j \leq k \leq d$ and $u_{1}$ as the source node $\mathrm{u}$, as $u_{k+1}$ the destination node $v, \mathrm{k}$ for routing hop, $\mathrm{d}$ for the network diameter. Here, the network diameter $d$ is defined as the maximum hop count between any two nodes in the network.

Set up more power level after routing, starting from the source node $\mathrm{u}$, nodes according to the routing rules 1 and 2 to determine the route to the destination node $\mathrm{v}$, the process can be expressed as

$$
u_{1} \stackrel{u_{2}}{\rightarrow} v, u_{2} \stackrel{u_{3}}{\rightarrow} v, \cdots, u_{k-1} \stackrel{u_{k}}{\rightarrow} v, u_{k} \stackrel{v}{\rightarrow} v
$$

Where $u_{1} \subset R T_{\min p l}^{\left(u_{1}, v\right)}, \quad u_{2} \subset R T_{\min p l}^{\left(u_{1}, v\right)}, \quad u_{3} \subset R T_{\min p l}^{\left(u_{2}, v\right)}, \cdots \cdots \cdots, u_{k-1} \subset R T_{\min p l}^{\left(u_{k-1}, v\right)}, \quad u_{k} \subset R T_{\min p l}^{\left(u_{k}, v\right)}$, $u_{k+1} \subset R T_{\min p l}^{\left(u_{k}, v\right)}$.

$$
\text { And } p l_{\text {min }}\left(u_{1}, u_{2}\right) \geq p l_{\text {min }}\left(u_{2}, u_{3}\right) \geq L \geq p l_{\text {min }}\left(u_{k-2}, u_{k-1}\right) \geq p l_{\text {min }}\left(u_{k-1}, u_{k}\right) \geq p l_{\text {min }}\left(u_{k}, v\right) \text {. }
$$

In this way, so as to form a $u$ from the source node to destination node v power level minimum and not increasing the routing.

Step 4 of the practice of data packet and MAC layer control frame power control: according to their activities on the routing node to transmit packet routing tables, the power level of sending data packet in accordance with the routing pt_null the power level Settings, namely

$$
p l_{\text {Data }}\left(u_{j}, u_{j+1}\right)=p l
$$

Among them, $p l$ the power level for the routing $u_{j}$ table.

At the same time $u_{j}$ set the transfer corresponding MAC layer control frame power level $p l_{M A C}$ consistent with $p l$, that is

$$
p l_{M A C}\left(u_{j}, u_{j+1}\right)=p l
$$

PC - only to effective AODV routing maintenance, routing maintenance, like AODV, using the Hello grouping and RERR (Route ERRor) group. The process is different from AODV is to: (1) when the node monitoring on the route to the routing is not available, will inform $u$ the source node routing repair; (2) node in send Hello grouping and RERR packet sending power level consistent with the existing effective an item from a routing table $p l$, the corresponding control at the MAC layer frame send power level $p l_{M A C}$ consistent with $p l$.

\section{Performance Analysis}

Set $p(u, v)=u u_{1}, \cdots, u_{k-1} u_{k} v$ said from node $\mathrm{u}$ to node $\mathrm{v}$ a undirected path, expressed as a $\mathrm{n}$ network perform routing lookup power series, $\mathrm{N}$ as the number of network nodes, and so have the following theorem.

Theorem 1 the time complexity of the algorithm $o(3 d \times n-d)$. 
Demonstrates that in the worst case, a power level network node $\mathrm{u}$ send RREQ packet along the path $p(u, v)$, time complexity for $\mathrm{d}$ orders of magnitude. Node $\mathrm{v}$ sends RREP packet back along the path $p(u, v)$ at the same time, the time complexity for $\mathrm{d}$ orders of magnitude. Here said the network diameter $\mathrm{d}$, node $\mathrm{u}$ and $\mathrm{v}$ to transmit the routing packet round-trip time complexity $2 d \times n$ for $2 \mathrm{~d}$ orders of magnitude. Algorithm were conducted $\mathrm{n}$ different power level search, its time complexity is orders of magnitude. The algorithm to choose a destination node power level minimum routing, its time complexity is orders of magnitude. So the total time complexity is a algorithm.

Theorem 2 communication complexity of the algorithm $o(2 N \times n)$.

Demonstrates that in the worst case, a power level network routing query distributed in $\mathrm{N}$ nodes at the same time, the communication complexity of the order of magnitude of $2 \mathrm{~N}$. Because the algorithm need to find $\mathrm{n}$ different power level, so the communication complexity of orders of $2 N \times n$ magnitude, that is for $o(2 N \times n)$.

Theorem 3 routing algorithm is no loop routing.

Proof: assumes that there is a circular route is shown in Figure 1, figure in the dashed lines between nodes exist multiple hops. If $\mathrm{u}$ Source node to the destination node $\mathrm{v}$ path $\mathrm{b}-\mathrm{c}-\mathrm{e}-\mathrm{f}$ - $\mathrm{g}$ - $\mathrm{b}$ cycle, which means that the routing message after a node and then returns to the node $\mathrm{b}$ $\mathrm{b}$, path $\mathrm{b}, \mathrm{c}$ and other node receives the same routing request for many times, this and the algorithm to the < Source ID, Broadcast ID, $\mathrm{pl}>$ determine whether receive this by request, if again receive it discards chain by request contradict each other. So the routing algorithm is no loop routing.

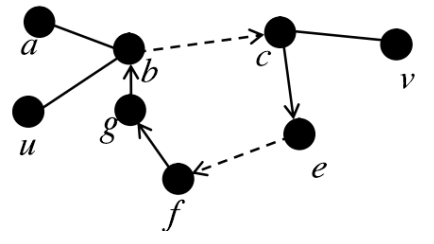

Figure 1. Exist Loop Routing

PC - AODV routing, according to the need to build more power level node, in turn, select effective route to the destination node power level minimum, at the same time in the transmitting data packet, routing and MAC layer control message frame in the process of adjust the transmission power level with consistent, which can effectively reduce the energy consumption. According to the routing rules 1 and 2, choose $p l=4$ the routing node $\mathrm{u}$ and node $\mathrm{i}$. Because node $\mathrm{j}$ and $\mathrm{k}$ retains the route to the destination node $\mathrm{v}$, respectively, according to the routing rules 1 and 2 , nodes choose node $\mathrm{k} \mathrm{j}$, k node selection $j \stackrel{p l=3}{\leftrightarrow} \stackrel{p l=3}{\leftrightarrow}^{p} v$ to the destination node $\mathrm{v}$. Then node $\mathrm{u}, \mathrm{i}, \mathrm{j}$ and $\mathrm{k}$, respectively to adjust the transmission power level to $4,4,3$, and 3, along the respective valid routing to transmit data packet and MAC control frame to the next hop $k \stackrel{p l=3}{\leftrightarrow} v$. Thus, the PC - AODV protocol is helpful to reduce the network energy consumption.

\section{Simulation Results}


In order to effectively evaluate the CPC - the performance of AODV, this chapter using the Network simulation software NS2 (Network Simulator) simulation comparison. For the simulation area, random distribution in this area 100 nodes, each node initial energy for $20 \mathrm{j}$, packet arrival as Poisson flow, service time is exponential distribution. Assume that each node can change the transmission power, and changes in power level is only five. Table 1 shows the detailed simulation parameters, through these parameters will be PC - AODV and CLUSTERPOW, AODV and TPR (Topology - -based Power - aware Routing).

Table 1. The Simulation Parameters

\begin{tabular}{|c|c|}
\hline Parameter & Value \\
\hline NS version & 2.3 \\
\hline Number of nodes & 100 \\
\hline Mobility model & Random way point \\
\hline Terrain range $\left(\mathrm{m}^{2}\right)$ & $1000 \times 1000$ \\
\hline Propagation model & Two-ray ground reflection \\
\hline Number of transmit power levels & 5 \\
\hline $\begin{array}{l}\text { Ranges corresponding to the } \\
\qquad \mathrm{pl}(\mathrm{m})\end{array}$ & $90,130,170,210,250$ \\
\hline Average node degree & 5 \\
\hline Node's mobility speed (m/s) & $0-20$ \\
\hline Rate of channel (Mbps) & 2 \\
\hline Type of traffic & CBR \\
\hline Number of FTP flows & $10-36$ \\
\hline Packet size (Bytes) & 1400 \\
\hline MAC & IEEE 802.11 \\
\hline Simulation time (s) & 1000 \\
\hline
\end{tabular}

On experiments, randomly selected from the source node and destination node, mainly from the following aspects to evaluate the characteristics of PC - AODV.

Routing overhead (routing overhead) : unit time send the number of routing control group. Packet delivery ratio (packet delivery thewire) : all the destination node receives the success on the number of data packet and its corresponding source node grouping of data sent to the destination node number of the ratio of the average. Average end-to-end delay (business, end - to - end delay) : data packet from a to the destination node receives the right of the average time, which include the queue delay of intermediate nodes, MAC layer retransmission time and more intermediate nodes forward time, etc. In different literature, researchers on network life (network lifetime) definition, this paper adopts the definition of literature (68-69), which is defined as: network life: first in the network energy consumption due to communication up its existing node. 
In the following simulation experiment, each scenario simulation run 50 times, each point in the curve of numerical simulation run is the average of the total. Figure 2 shows under different network load on average, four routing protocol routing overhead performance curve. With the increase of average load of network, four kinds of the average routing protocol routing overhead will increase, in contrast with PC CLUSTERPOW increase sooner - AODV, followed by AODV, the smallest is TPR. CLUSTERPOW in the same situation with PC AODV is AODV and TPR, added 22 KBPS - 35 KBPS routing overhead. This is because the CLUSTERPOW established different with PC - AODV were more power level routing, and AODV and TPR only to establish a power stage routing. CPC - AODV routing overhead is relatively less CLUSTERPOW $10 \mathrm{KBPS}$ - $55 \mathrm{KBPS}$, this is because the latter to establish more active power level routing, and CPC - AODV is according to the need to build more power level routing. AODV routing overhead than TPR KBPS 2-7 KBPS, this is because the TPR in AODV, on the basis of the introduction of optimization neighbor set to reduce routing flooding.

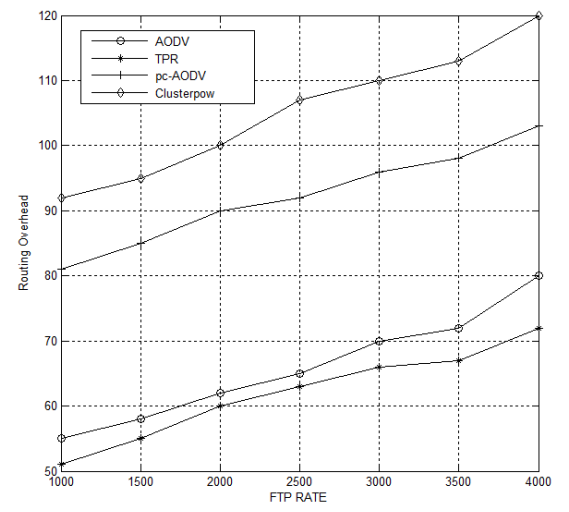

Figure 2. Different Network Load

Figure 3 said under different network load on average, four contrasting network routing protocol. As you can see from figure 3: with the increase of average load of network, four routing protocols of network life will reduce gradually, AODV decreases more quickly, the second is CLUSTERPOW with TPR, under the condition of same PC - AODV network change the minimum service life. This is because the AODV power control measures were not taken, using the default maximum power to transmit data, pass the same data will consume more energy, and the other three agreements are considering the energy consumption of communication. TPR and CLUSTERPOW performance in terms of network lifetime, and the comparison, PC - AODV combination of MAC layer power control to further reduce the network energy consumption, energy saving of $12 \%$ on average. With the increase of the average load on the Internet, the network energy consumption will increase, some of the large flow in the AODV node will run out of energy, the corresponding residual energy is less, the second is CLUSTERPOW and TPR. Obviously with the increase of the average network load, PC - AODV can save the network energy consumption, and can prolong network lifetime. 


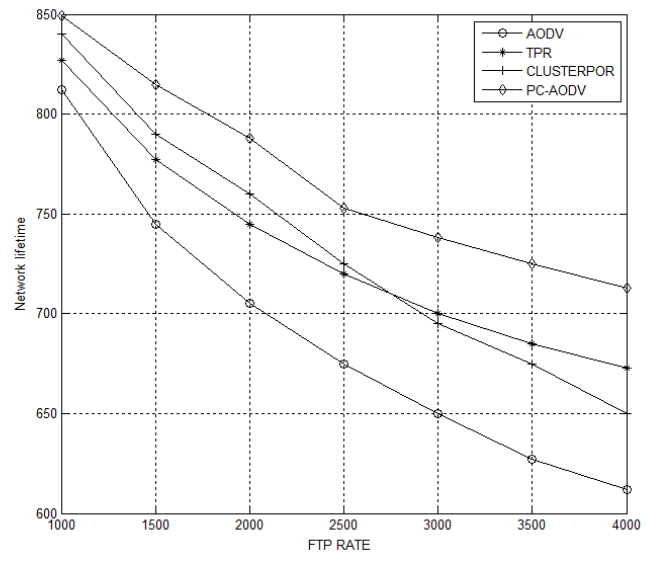

Figure 3. Network Lifetime

\section{Conclusion}

Introduction of power control in Ad Hoc network routing protocol can not only reduce the network energy consumption, but also can improve the network throughput, delivery rate, such as performance, has become the current mobile Ad Hoc network is a research hot spot. This paper puts forward a kind of on-demand routing protocol based on cross-layer power control PC - AODV. PC - AODV on-demand build several different power level routing, choose the way to the destination node with the minimum power level origin grouping, and the network layer of the data packet, routing messages and MAC layer control frame transmission using different power control strategy to reduce energy consumption. Research results show that the PC - AODV is helpful to reduce the communication overhead energy, prolong the network life, improve the delivery rate and improve the network delay.

\section{References}

[1] G. Zhao, X. Q. Liu and M. T. Sun, "Energy-aware geographic routing for sensor networks with randomly shifted anchors", Proceedings of 2007 IEEE Wireless Communications and Networking Conference (WCNC 2007), Hong Kong, China, (2007), pp. 3456-3461.

[2] G. Zhao, X. Q. Liu and M. T. Sun, "Energy-efficient geographic routing with virtual anchors based on projection distance", Computer Communications, vol. 31, no. 10, (2008), pp. 2195-2204.

[3] S. B. Wu and K. S. Candan, "GPER: geographic power efficient routing in sensor networks", Proceedings of the 12th IEEE International Conference on Network Protocols (ICNP 2004),Berlin, Germany, (2004), pp. $161-172$.

[4] S. B. Wu and K. S. Candan, "Power-aware single and multipath geographic routing in sensornetworks", Ad Hoc Networks, vol. 5, no. 7, (2006), pp. 974-997.

[5] R. H. Wendi, C. Anantha and B. Hari, "Energy-efficient communication protocol for wirelessmicrosensor networks", Proceedings of the 33rd Annual Hawaii International Conference onSystem Sciences (HICSS33), Hawaii, USA, (2000), pp. 3005-3014.

[6] O. Younis and S. Fahmy, "HEED: a hybrid, energy-efficient, distributed clustering approach for adhoc sensor networks", IEEE Transcations on Mobile Computing, vol. 3, no. 4, (2004), pp. 660-669.

[7] L. M. Feeney and M. Nilsson, "Investigating the energy consumption of a wireless network interface in an ad hoc networking environment", Proceedings of the the 20th IEEE International Conference on Computer Communications (INFOCOM 2001), Alaska, USA, vol. 3, (2001), pp. 1548-1558.

[8] W. Zhang, H. He, Q. Zhang and T.-H. Kim, "PhoneProtector: Protecting User Privacy on the Android-Based Mobile Platform", International Journal of Distributed Sensor Networks, Article ID 282417, vol. 2014.

[9] C. W. Tan and S. K. Bose, "Modifying AODV for efficient power-aware routing in MANETs", Proceedings of 2005 ACM/Kluwer Mobile Networks and Applications (TENCON 2005), Melbourne, Australia, (2005), pp. 1-6. 
[10] S. H. Lee, E. Choi and D. H. Cho, "Timer-based broadcasting for power-aware routing inpower-controlled wireless ad hoc networks", Communications Letters, vol. 9, no. 3, (2005), pp. 222-224.

[11] K. Woo, C. Yu and D. Lee, "Non-blocking localized routing algorithm for balanced energyconsumption in mobile ad hoc networks", IEEE International Workshop on Modeling, Analysis \& Simulation of Computer and Telecommunication Systems 2001 (MASCOTS 2001), Ohio, USA, (2001), pp. 117-124.

[12] M. J. Hossain, M. A. Dewan and O. Chae, "Maximizing the effective lifetime of mobile ad hoc networks", IEICE Transactions on Communications, vol. 91, no. 9, (2008), pp. 2818-2827.

[13] A. Sankar and Z. Liu, "Maximum lifetime routing in wireless ad-hoc networks", Proceedings of the23rd IEEE International Conference on Computer Communications (INFOCOM 2004), HongKong, China, vol. 1, no. 2, (2004), pp. 1089-1097.

[14] W. B. Heinzelman, A. P. Chandrakasan and H. Balakrishnan, "An application-specific protocol architecture for wireless micro sensor networks", IEEE Trans, on Wireless Communications, vol. 1, (2002) October, pp. 660-670.

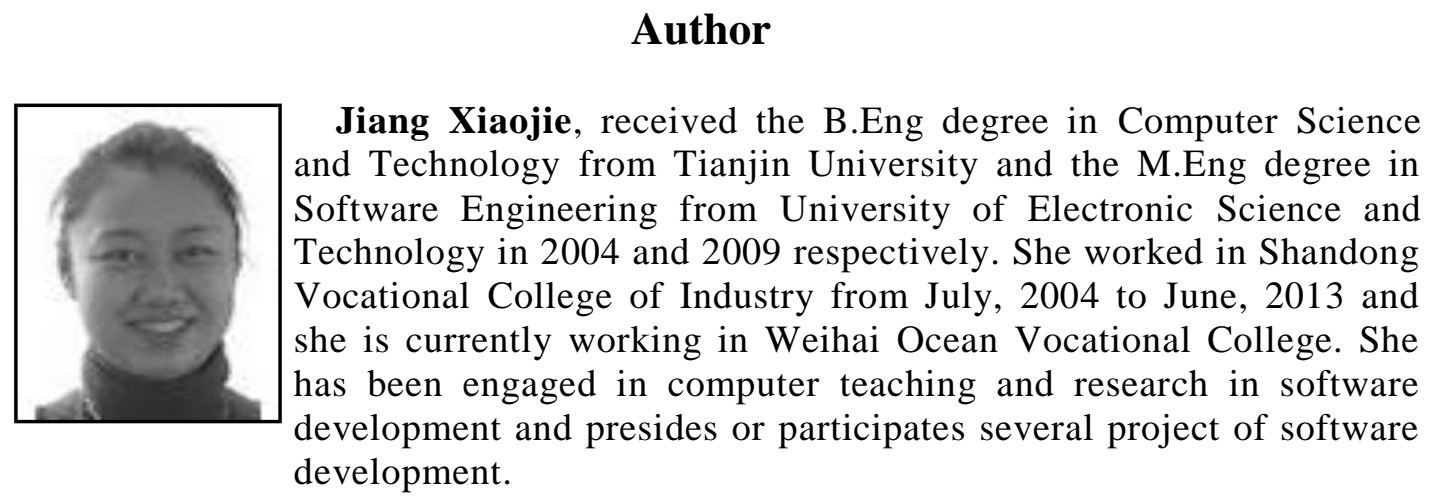

